\section{Pulmonary scedosporium infection as a complication of infliximab therapy for ankylosing spondylitis}

A 47-year-old man with a negative Mantoux test received infliximab $800 \mathrm{mg}$ fortnightly in August 2005 for treatment of ankylosing spondylitis. He developed haemoptysis after receiving the third dose in September 2005 and therefore infliximab was stopped. CT thorax in April 2006 showed evidence of mycetoma at the left upper lobe (fig 1A, B). In view of persistent haemoptysis and radiological deterioration, voriconazole $200 \mathrm{mg}$ daily was started and left upper lobe lobectomy was performed. Histopathology showed caseous granulomatous inflammation, destroyed bronchial wall with inflammatory cell infiltration and fungal mycelium was seen. Culture of the resected tissue grew Scedosporium apiospermum (fig 2A, B). Haemoptysis subsided after surgery.

\section{J C Ngai, ${ }^{1}$ R Lam, ${ }^{2}$ F W Ko, ${ }^{1}$ K-W To, ${ }^{1}$ D S Hui ${ }^{1}$}

${ }^{1}$ Department of Medicine and Therapeutics, The Chinese University of Hong Kong, Prince of Wales Hospital, Shatin, New Territories, Hong Kong; ${ }^{2}$ Department of Microbiology, The Chinese University of Hong Kong, Prince of Wales Hospital, Shatin, New Territories, Hong Kong
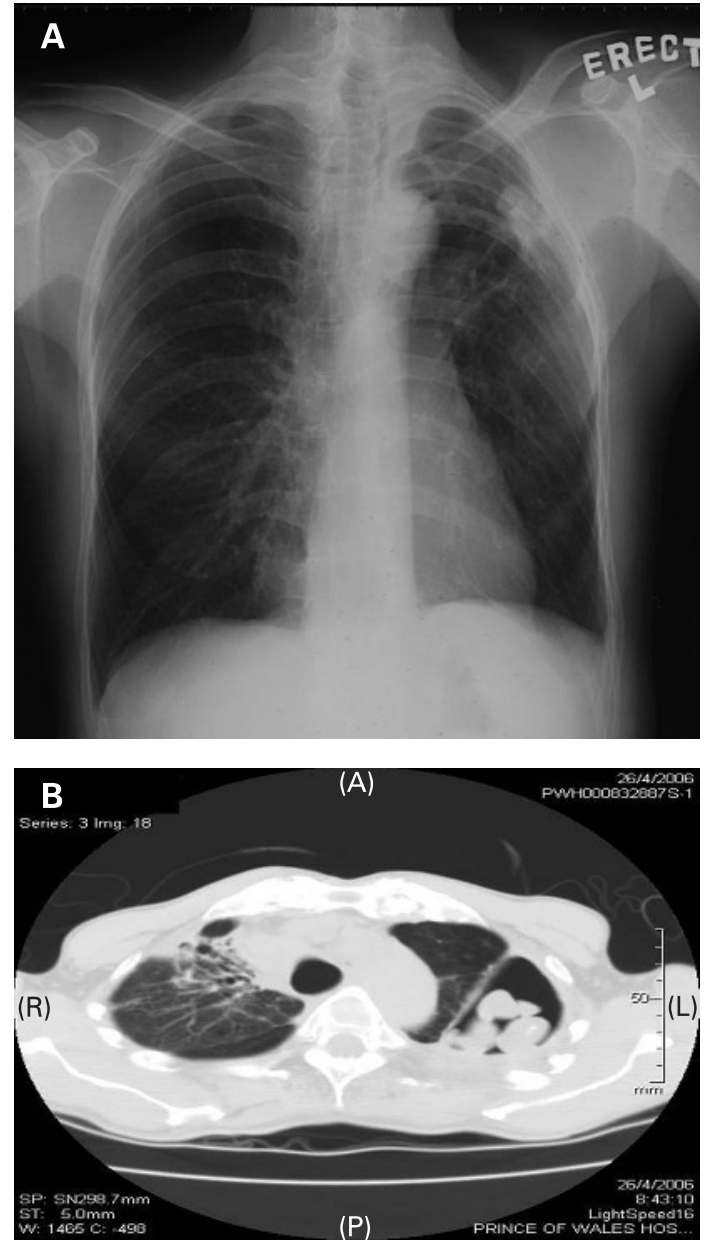

Figure 1 (A) Chest $x$ ray showed a small and non-specific opacity at the left upper lobe (LUL). His chest $x$ ray prior to commencement of infliximab was unremarkable. (B) Chest CT revealed a cavity at the posterolateral aspect of LUL with several opacities within, suggestive of mycetoma. Inflammatory changes were also noted at the right upper lobe.
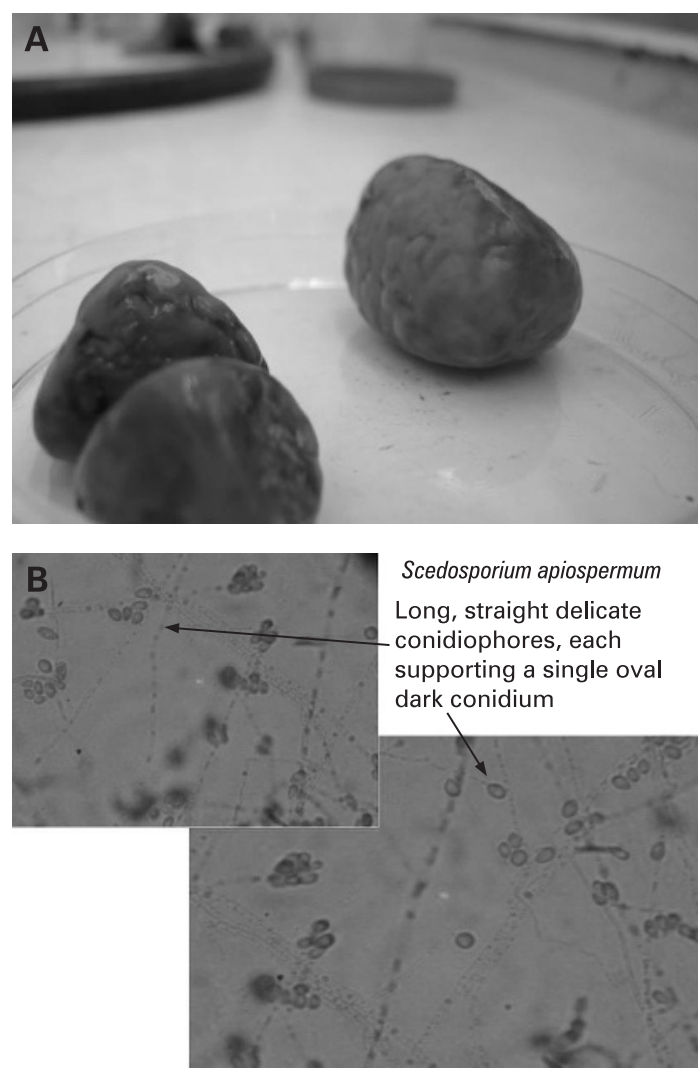

Figure 2 (A) Three fungal balls removed from the left upper lobe cavity. (B) Scedosporium apiospermum is the species of hyaline molds commonly encountered in clinical practice. Microscopically, hyaline mold is characterised by the formation of conidia singly from the tip of conidiophores. The conidiophores of $S$ apiospermum are straight and narrow with no swelling. Some conidia aggregated into bundles in the background.

\section{Learning points:}

- Scedosporium infection may present in a wide range of pulmonary manifestations from simple colonisation to mycetoma formation and invasive disease, simulating aspergillosis. ${ }^{1}$

- Antitumour necrosis factor agents should be used with caution as these may lead to opportunistic infections as a result of suppression of T cell mediated immunity. ${ }^{2}$ To the best of our knowledge, this is the first case report of pulmonary scedosporium infection as a complication of infliximab therapy for ankylosing spondylitis.

Correspondence to: Dr D S Hui, Department of Medicine and Therapeutics, The Chinese University of Hong Kong, Prince of Wales Hospital, 30-32 Ngan Shing St, Shatin, New Territories, Hong Kong; dschui@cuhk.edu.hk

Competing interests: None.

Ethics approval: Ethics approval was obtained.

Patient consent: Obtained.

Accepted 5 March 2008

Thorax 2009;64:184. doi:10.1136/thx.2008.097626

\section{REFERENCES}

1. Koga T, Kitajima T, Tanaka R, et al. Chronic pulmonary scedosporiosis simulating aspergillosis. Respirology 2005:10:682-4.

2. Cohen RD, Bowie WR, Enns R, et al. Pulmonary actinomycosis complicating infliximab therapy for Crohn's disease. Thorax 2007:62:1013-14. 